\title{
Design and Analysis of Ultrasonic Horns Operating in Multiple Vibration Modes
}

\author{
Ziad Shakeeb Al Sarraf \\ Department of Mechanical \& Mechatronics Engineering, Faculty of Engineering, \\ University of Mosul, Mosul, Iraq
}

\begin{abstract}
A number of recent studies have shown that combining different modal responses can provide opportunities to improve the vibration behaviour of the output faces of tuned ultrasonic horns to provide a more effective use of the ultrasonic energy. Investigation the benefits of combining different modal responses with a view to optimizing the energy transfer from a range of power ultrasonic devices that rely on tuned horns is essential. This research will therefore aim to investigate the use of combining and exciting different vibration modes in order to design more effective resonant horns for use in high power ultrasonics applications such as metal forming, welding, cleaning and surgical devices. The research is extended to study the possibility of design an ultrasonic transducer which can operate in multiple vibration modes by modify its geometric features. The longitudinal- torsional mode is selected first because of its wide applications in ultrasonic field. The effect of geometrical modifications of transducer's matching part is being analyzed analytically, numerically and experimentally. The suggested modifications are including cut of slots and reduce the cross sectional area so that the excited longitudinal vibrational mode can be regenerated into a longitudinal-torsional mode. The considerations of simplicity of manufacturing and exciting and the efficient of energy conversion are the main advantages of the proposed transducer.
\end{abstract}

Keywords-ultrasonic transducer, design horn; resonant frequency; model and harmonic analysis

DOI: $10.7176 / \mathrm{JIEA} / 9-3-02$

Publication date:May $31^{\text {st }} 2019$

\section{Introduction}

Ultrasonic transducer is a device which receives electrical energy from a power supply and converts it into a vibrational motion; based on how the particles move in the guide, there are three principle modes of the vibration motion which are namely longitudinal, torsional and flexural modes. With the development of ultrasonic power applications especially in motoring, drilling and cutting processes, the needs for coupling of these modes to produce new modes is being essential. One of the important modes is the longitudinal-torsional mode. There are two main methods to produce this mode in ultrasonic systems, either by coupling of longitudinal and torsional modes so that they resonate at the same frequency [1] or by degenerating of longitudinal mode to produce the desired mode [2]. Coupling of modes has many disadvantages which are the need for using two electric generators, the need to use an expensive piezoceramics which polarized in radial mode to generate the torsional mode [3] and the difficulty to hold the transducer within an enclosure because the positions of motional nodes of these two modes along the device are different [4]. Degeneration of modes is more applicable, inexpensive manufacture, need only longitudinal excitation and easy to fix into an enclosure using the longitudinal nodal position, however; the low efficiency of mode conversion is the main disadvantage of this method $[1,9]$.

There are many attempts to model the longitudinal-torsional mode in ultrasonic systems to improve the conversion performance, Lin [1] performed an analytical model of compound modes transducer which used two sets of piezoceramic rings, the first set is polarized in thickness direction to generate the longitudinal mode and the second set is polarized in radial direction to generate the torsional mode, and by using exponential solid horn as matching part of the transducer, the two modes are combined to produced the desired mode, he used Mason's equivalent circuit by deriving the acoustic impedance equations of each part and then solve for the whole transducer, the consideration of equal resonance frequency of both longitudinal and torsional modes is used to extract the other design parameters. Liu [11] revealed a study of hybrid longitudinal-torsional vibrator driven by two longitudinal ultrasonic transducers which is proposed for ultrasonic-assisted wire drawing. However, the described model used two sets of piezoceramic to generate these modes which referred to be an expensive method and also the mechanical and electrical losses are not included in that model. Qi [3] modelled an ultrasonic micro motor using the equivalent circuit method, the damping effects is considered as an electric resistance in the circuit and its value driven from the overall quality factor of the system, the model is simplified as only has longitudinal response and then an assumption of linear relationship between the torsional and longitudinal velocities at the output surface are considered, the analytical model validated by an experimental model with good agreement. Harkness [2] is employed the finite element model to study two design techniques for obtaining longitudinal torsional vibration in ultrasonic step horns, the model is validated by an experimental analysis and showed a good agreement. 
In this study, a new designed Langevin transducer which employs geometrical modifications on transducer front mass to regenerate of longitudinal vibration produced in piezoceramic stack into longitudinal-torsional vibration on the outer surface is analyzed. Numerical, Analytical and experimental models are employed to study the performance of transducer under low and high excitation voltages. The mechanical losses are considered in the analytical and numerical models and showed good improvement for these models as compared with the experimental model.

\section{Numerical Model of transducer}

Numerical analysis is being widely used to design transducers; its suitability comes from the ability to describe principle modes of vibration and the coupling between these modes besides the capability to model complex geometry shapes. A FE software package ABAQUS is implemented for this analysis.

The study is started with analyzing the effect of cutting slots along a uniform bar (horn) on the mode coupling, then the model is developed by reduce the cross section area exponentially along the bar; the optimized horn is then used as matching part in a traditional Langevin transducer. Figure 1 shows the transducer structure which is composed of two piezoceramic disks sandwiched between two masses and assembled by a titanium central bolt. The front mass is an exponential decade bar made from titanium and spirally slotted along its length. The back mass is cylindrical and made from steel, transducer materials are chosen such that the acoustic impedances of the front and back masses match that of the piezoceramic material and therefore minimize internal reflection [5]. All parts' dimensions are taken relative to the piezoceramic disk thickness so that the model can be scaled and tested for different sizes based on the required application, a different piezoceramic disks thickness are studied which range between (1-10) $\mathrm{mm}$ so that the total length of modelled transducers are range between (26-260) $\mathrm{mm}$. One of these models is then selected to be analytically analyzed and fabricated; the selected transducer has $5 \mathrm{~mm}$ piezoceramic disk thickness.

A modal analysis has been first performed in FE analysis to extract the natural resonance frequencies and the corresponding modal shapes of the transducers, and then a harmonic analysis is done by applying different excitation voltages. The longitudinal and torsional response of a point on the circumferential of transducer out surface has been extracted. The internal structural losses of the transducer have been considered by applying global damping using the Rayleigh formula, the viscous constant $\beta$ being defined by the following expression:

$$
\beta=\frac{1}{\omega_{r} Q_{m}} \text {. }
$$

Where $\omega_{r}$ is the modal frequency and $Q_{m}$ is the overall mechanical quality factor which is estimated at the beginning of the analysis and then corrected after and experimental harmonic analysis of the fabricated transducer.

Although it is possible to model the piezoelectric effects and the mechanical losses in FE model, there are other parameters which influenced the dynamic behaviour of the transducer but could not be included in the numerical model. These parameters are the electric and electric-mechanical losses effects, the pre tress effects on resonance and responses and finally the load impedance effects, therefore; there is a need to create an analytical model which can deeply analyze these effects.

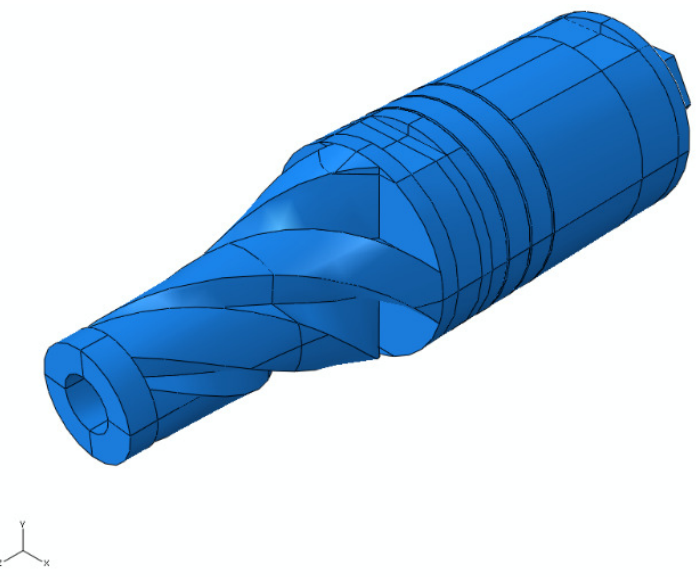

Figure 1. Finite element transducer

\section{Model Analysis}

Analytical solution to the wave equation in piezoelectric transducers is difficult to derive from first principles, difficulties comes from the complexity in geometry of transducer parts, the usage of different materials, coupling 
of electrical and mechanical effects and the difficulty in represent of joints and fasting between parts [6]. However, there still an ability to adopt analytical model using electro-mechanical equivalent circuit which is based on onedimensional elastic wave theory. The similarity between mechanical vibration and electrical resonance makes the electromechanical equivalent method an efficient way to deal with ultrasonic transducer design. Mason model presented an exact equivalent circuit that represents the transducer parts as an impedance T-network circuit. The model has been widely used for modelling loaded and unloaded transducers [7,8]. Figure 2 shows the equivalent circuit of the selected transducer, the transducer is divided into six regions with front and back load impedances. Regions two and three are contain two different parts' sections which are the back mass and the pre stress bolt in region two and the piezoelectric stack with electrodes and the pre stress bolt in region three, in order to find the mechanical impedances of these regions, there are two assumptions, the first assumption is used the average values of parts properties (density and modulus of elasticity) to form the mechanical impedance of the regions [7]. The second assumption is considered a constant strain in both parts of the region and therefore the acoustic elements should be added in series [8], both of these assumptions are used for comparison. The mechanical impedance of non uniform cross sectional area of the front mass in region 5 is assumed to be as an exponential horn with longitudinal slots because the solution considers only the longitudinal vibration, its impedance is computed as a function of position along its length [10]. The impedance of front and back loads (to air, as the transducer is tested under unloaded condition) is assumed to be zero (short circuit). Also the losses of mechanical parts have been represented using complex material constants where the imaginary parts represent the losses which are taken from the quality factors of the used materials [5]. The longitudinal velocity response at the outer surface of transducer can be found under different excitation voltages using the current equation pass through the right impedance $\left(Z_{6}\right)$ of region 6 (I9), the torsional velocity response is assumed to be in a linear relationship with the longitudinal velocity response, this assumption will be proved by other models.

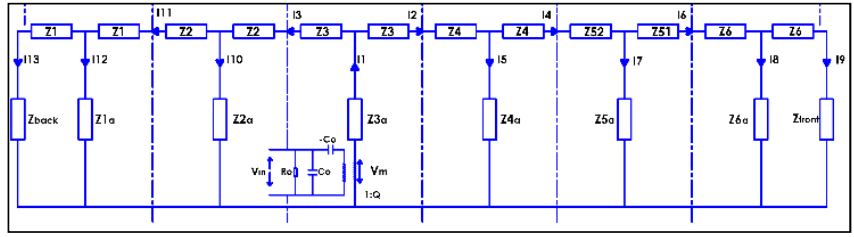

Figure 2. Equivalent circuit of transducer.

\section{Experimental Analysis}

The fabricated transducer is shown in Figure 3. The piezoceramic disk thickness is $5 \mathrm{~mm}$ and the overall length of the transducer is $130.5 \mathrm{~mm}$. Two experimental tests are carried out; the first test is an impedance analysis test using Agilent (4294A Precision Impedance Analyzer) to validate the theoretical model described above, the second experimental is a swept sine analysis using a Laser Doppler Vibrometer (Polytec CLV 3D laser vibrometer) to measure the longitudinal and torsional velocity responses of a point on the circumference of transducer outer face. The responses are measured for a range of excitations and the results are compared with the numerical analyses.

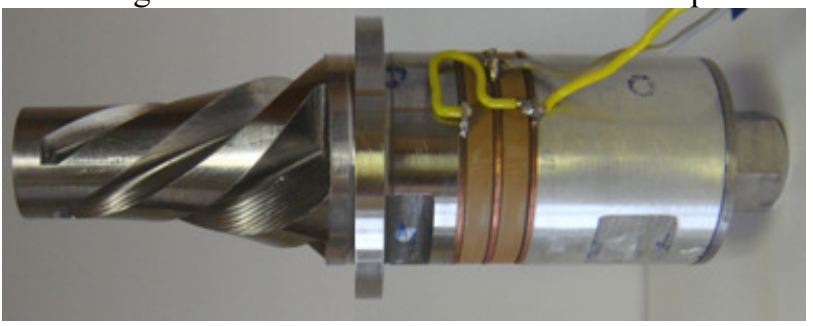

Figure 3. The fabrication of ultrasonic transducer

\section{Results and Discussion}

Figure 4 shows the impedance magnitude of the analytical and experimental analyses. There is a difference in impedance magnitudes and resonance frequencies between the analytical and experimental results; the difference is may caused by a number of factors. First, the material property may be different for the standard and actual values of the transducer material, second, the equivalent circuit model is an approximate model and therefore it neglects many practical factors, and finally, the transducer has a complex structure, especially the part with spiral structure and its modelling is more complex. Also the effect of central bolt pre stress which influenced the resonance frequency is not included. However, the difference in frequency within $2 \%$ for resonance and $4 \%$ for anti-resonance region, and therefore it could be considered for prediction of transducer resonance region prior to fabrication.

The harmonic analysis results of fabricated transducer are shown with the numerical results in Figure 5, where the peak responses at resonance and corresponding torsionalities are shown for different excitation voltages. The 
nonlinear increase in transducer responses with increase of voltages does not affect on the torsionality which is almost constant; this nonlinearity is due to increase of dielectric losses with increase of excitation which is not considered in numerical model, also the relative motion between transducers' parts at high excitation leads to increase of damping, whereas it is considered a constant damping over excitation range in FE model. The torsionality made by the FE analysis is over predicted the experimental results at all excitation voltages, this may be related to the internal structure of the transducer, the central bolt providing less torsional than longitudinal stiffness, whereas in the FE model, the boundary conditions are simple constrains in both directions. Less torsional stiffness, and potential torsional movement between mechanical elements during operation, could be responsible for additional torsional damping and the underperformance of the mechanical system.

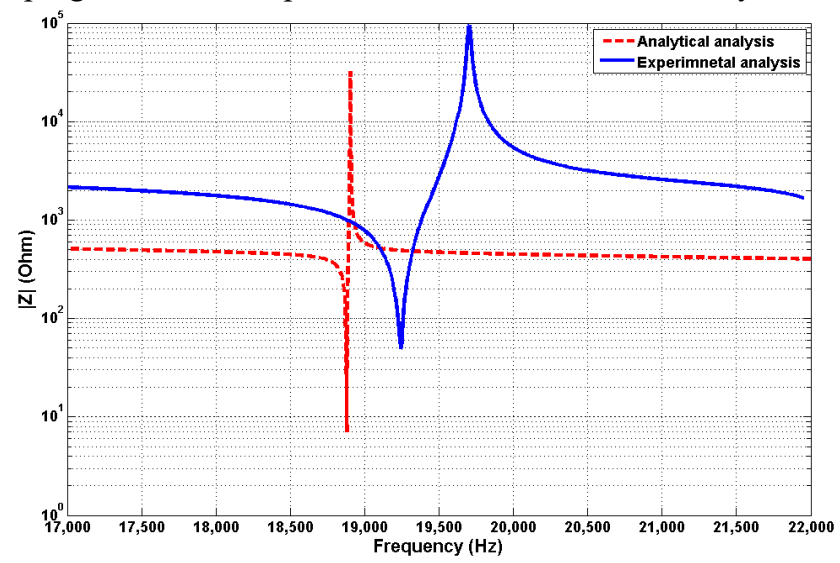

Figure 4. Impedance curve from analytical and experimental

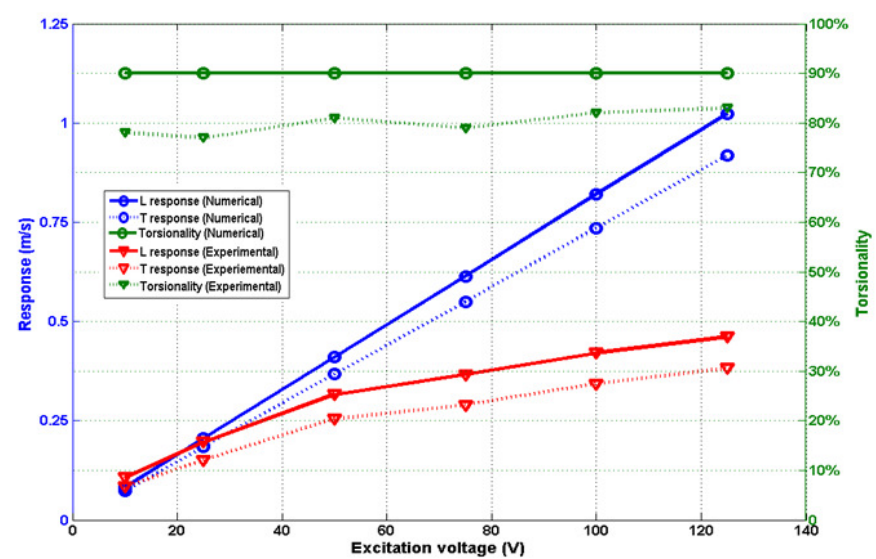

Figure 5. Numerical and experimental peak responses and torsionality for different excitation voltages.

\section{Conclusion}

Numerical, analytical and experimental models are designed to study the dynamic characteristics of proposed longitudinal-torsional ultrasonic transducer. Combining of these modes could overcome the difficulty of study the effects of different parameters/ factors on the transducer dynamic performance. The effect of material losses are involved and led to improve the numerical and analytical models respectively. Mode degeneration technique is proved to be the practical method to produce high torsionality transducers with high responsiveness and good range of operation frequencies.

\section{References}

[1] S. Lin: Sandwiched Piezoelectric Ultrasonic Transducers of Longitudinal-torsional Compound Vibrational Modes, Ultrasonics, Vol. 44(6), (1997), p. 1189-1197.

[2] P. Harkness, A. Cardoni and M. Lucas: Ultrasonic Rock Drilling Devices Using Longitudinal-Torsional Compound Vibration, IEEE International Ultrasonic Symposium, Rome, Italy (2009).

[3] A. Qi, J. Friend and L. Yeo: An Analytical Model for a Twisted Beam Piezoelectric Ultrasonic Micromotor, 5th Australasian Congress Symposium on Appl. Mech., Birsbane, Australia (2007).

[4] Wuchinich: Longitudinal-Torsional Ultrasonic Tissue Dissection, U.S. Patent, No. US 7,762,979 B2, United States (2010).

[5] R. Mahdavinejad: Finite Element Dimensional Design and Modeling of an Ultrasonic Transducer, Iranian 
Journal of Science \& Technology, Transaction B, Engineering, 29(B2), (2005).

[6] S. Sherrit, S. Leary and Y. Bar-Cohen: Comparison of the Mason and KLM Equivalent Circuits for Piezoelectric Resonators in the Thickness Mode, IEEE International Ultrasonics Symposium,( 1999).

[7] T. Li, Y. Chen, J. Ma: Development of a Miniaturized Piezoelectric Ultrasonic Transduce, IEEE Ultrasonics, Vol. 56(3), (2009), p. 649-659.

[8] S. Sherrit, B. Dolgin, and Y. Bar-Cohen: Modeling of Horns for Sonic/Ultrasonic Applications, IEEE Ultrasonics Symposium, (1999).

[9] Karafi M, Hojjat Y and Sassani F. A new hybrid longitudinal- torsional magnetostrictive ultrasonic transducer. Smart Mater Struct 2013; 22: 1866.

[10] Chen T, Liu S, Liu W and Wu C. Study on a longitudinal-torsionalultrasonic vibration system with diagonal slits. Advances in Mechanical Engineering 2017; Vol. 9(7), p. 1-10.

[11] Liu S, Shan X, Guo K, and Xie T. Design and Fabrication of a Skew-TypedLongitudinal-Torsional Composite Ultrasonic Vibrator for Titanium Wire Drawing. IEEE Access 2016; Vol.4, p. 6749-6755. 